\title{
First principle analysis of Coandă Micro Air Vehicle aerodynamic forces for preliminary sizing
}

\begin{abstract}
Purpose - The purpose of this paper is to reformulate the governing equations incorporating major variables and parameters for the design a Micro Air Vehicle (MAV), to meet the desired mission and design requirements. Design/methodology/approach - Mathematical models for various spherical and cylindrical Coanda MAV configurations were rederived from first principles, and the performance measures were defined. To verify the theoretical prediction to a certain extent, a computational fluid dynamic (CFD) simulation for a Coanda MAV generic models was performed. Findings - The major variables and parameters of Coanda MAV have been formulated into practical guidelines, which relate the lift (or thrust) produced for certain input variables, particularly the Coanda MAV jet momentum coefficient. The influences of the geometrical parameters are elaborated. Research limitations/implications - The present analysis on Coanda jet-configured MAV is focused on the lift generation due to the Coanda jet effect through a meticulous analysis. The effects of viscosity, the Coanda jet thickness, the radius of curvature of the surface and the stability of Coanda jet are not considered and will be the subject of the following work. Practical implications - The results obtained can be used for sizing in the preliminary design of Coanda MAVs. Originality/value - Physical and mathematical models were developed which can describe the physical phenomena of the flow field near the Coanda MAV surfaces influenced by Coanda jet sheets and for obtaining a relationship between relevant variables and parameters to the lift of practical interest.
\end{abstract}

Keyword: VTOL; Circulation control; Coanda effect; Micro Air Vehicle (MAV); Unmanned air vehicle (UAV) 